\title{
Stochastic Bound on Delay for Guaranteed Rate Nodes
}

\author{
Milan Vojnović and Jean-Yves Le Boudec
}

\begin{abstract}
We find that the probabilistic bound on delay in the article by Goyal et al. in 1997. The problem originates from: 1) the difference between stationary and Palm probabilities, and 2) treating the arrival counting process over some random time intervals as if the time interval would be fixed. The error is propagated to some later work, e.g. recently by Bensaou $\mathrm{et} \mathrm{al.,}$ which derived some probabilistic delay bounds based on the work of Goyal et al. We give fixes to the above problem.
\end{abstract}

Keywords - Fair queueing, guaranteed rate clock, packet scheduling, quality of service, Palm probability.

\section{Introduction}

$\mathrm{I}_{\mathrm{N}}^{\mathrm{N}}$ N Goyal et al. [1], the authors derive a probabilistic bound on delay through a sequence of guaranteed rate (GR) nodes ${ }^{1}[3]$, under the assumption that the arrival process is with Exponentially Bounded Burstiness (EBB) [4]. In [2], the authors propose a credit-based fair scheduler, show that it belongs to the class of GR nodes, and then directly apply the probabilistic bound on delay found in [1]. More generally, it is known that many schedulers can be described as GR nodes, with appropriately defined rate and latency parameters. Further, the concept of GR node (recalled below) is a convenient way to abstract a complex system, such as a router or a subnetwork, which is made of schedulers and delay elements, work conserving or not (Chapter 2 in [5]). Thus, it is important to have delay bounds for GR nodes. The concept of GR node is, roughly speaking, equivalent to a service curve concept [5]. We show that the probabilistic bound on delay in [1] is incorrect (Theorem 4 therein). The error is propagated to Theorem 2 and Theorem 4 of [2]. We note that the reference [1] can be also found as [3], which suffers from the same problem.

We first introduce some notation and then explain where the problem comes from. We assume that the arrival of packets is described by a stationary marked point process $\left(T_{n}, L_{n}\right)_{n \in \mathbb{Z}}$, where $T_{n}$ is the arrival time of packet $n$ and $L_{n}$ its length in bits. For an interval $\mathcal{I} \subset \mathbb{R}$, let $A \mathcal{I}$ be the number of bits observed in $\mathcal{I}$, likewise, let $N \mathcal{I}$ be the number of packets in $\mathcal{I}$. We assume that packets are seen at the input of the system as instantaneous jumps of size equal to packet length. We assume that the point process of packet arrivals is a simple point process (i.e., for all $t, N\{t\} \in\{0,1\}$ ). Let $L^{\min }$ and $L^{\max }$ be the minimum and maximum packet length, respectively. We denote with $\mathrm{P}_{A}^{0}$ (resp. $\mathrm{P}_{N}^{0}$ ) the Palm probability with respect to $A$ (resp. $N$ ). Let $\mathrm{E}_{A}^{0}$ and $\mathrm{E}_{N}^{0}$ be the expectations with respect to $\mathrm{P}_{A}^{0}$ and $\mathrm{P}_{N}^{0}$. See [6] for an exposition of Palm calculus. Intuitively, one may

\footnotetext{
Manuscript received May 16, 2002. The associate editor coordinating the review of this letter and approving it for publication was Dr. J. Kim.

The authors are with EPFL, CH-1015 Lausanne, Switzerland (e-mail: milan.vojnovic@epfl.ch; leboudec@epfl.ch).

Publisher Item Identifier 10.1109/LCOMM.2002.804248.

${ }^{1}$ The original denomination in [3] is Guaranteed Rate Clock scheduler. We use the phrase "GR node" instead, as the GR property applies more generally to complete systems which cannot be defined as single schedulers.
}

think of $\mathrm{P}_{A}^{0}$ (resp. $\mathrm{P}_{N}^{0}$ ) as the probability of events as seen by an arbitrary bit arrival (resp. packet arrival).

We recall the definition of a GR node in [3]. A system is a GR node with rate $r$ and latency $e$ if it satisfies the following. First, there exists a sequence $\left(f_{n}\right)_{n \in \mathbb{Z}}$ (the "virtual finish times") which satisfies the recursion

$$
f_{n}=\max \left[f_{n-1}, T_{n}\right]+\frac{L_{n}}{r}, n \in \mathbb{Z},
$$

and such that for all $n \in \mathbb{Z}$, there exists some $m \leq n$ with $f_{m} \leq T_{m}$. Second, the departure time process $\left(T_{n}^{\prime}\right)_{n}$ satisfies $T_{n}^{\prime} \leq f_{n}+e$ for all $n$.

Note that our definition is slightly more general than the original definition in [1], in order to fit a stationary framework. It is not difficult to observe that the GR node definition is equivalent to saying that, for all $n \in \mathbb{Z}$, there exists some $m \leq n$ such that

$$
T_{n}^{\prime} \leq T_{m}+\frac{L_{m}+\cdots+L_{n}}{r}+e .
$$

$A$ is said to be with $(\lambda, C, c)$-EBB, if for all $\sigma \geq 0, s \leq t$,

$$
\mathrm{P}(A[s, t] \geq \lambda(t-s)+\sigma) \leq C e^{-c \sigma} .
$$

Note that the definition is with respect to $P$, the steady-state probability.

At some point (proof of Theorem 4 in [1]), the authors consider the following event. Fix some $n \in \mathbb{Z}$, then consider

$$
\left\{A\left[T_{N_{n}}, T_{n}\right] \geq \lambda\left(T_{n}-T_{N_{n}}\right)+\sigma\right\}
$$

where $N_{n}$ is the largest integer not greater than $n$, for which $V_{N_{n}-1}<T_{N_{n}}$. From the assumption that $A$ is with $(\lambda, C, c)$ $\mathrm{EBB}$, the authors in [1] conclude:

$$
\mathrm{P}\left(A\left[T_{N_{n}}, T_{n}\right] \geq \lambda\left(T_{n}-T_{N_{n}}\right)+\sigma\right) \leq C e^{-c \sigma} .
$$

However, we find that this does not follow from the EBB definition. The reason is twofold. First, the above event considers the number of bits observed over an interval, given there is an arrival at the boundary of the interval; thus the underlying probability is the Palm probability and not the stationary probability as given in EBB definition. Second, the length of the interval over which bits are observed is random, not fixed.

One may intuitively think of $A\left[T_{N_{n}}, T_{n}\right]$ as the number of bits one would observe if one picks up at random an arrival packet $n$, and then counts the number of bits observed since the beginning of the current busy period up to the time instant $T_{n}$. This is rigorously true if $e=0$ (then, $T_{N_{n}-1}^{\prime}<T_{N_{n}}$ ).

We show now that this methodological error has a fatal consequence on the validity of the final result in [[1], Theorem 4]. 
We do this by exhibiting an example where the delay bound in [1] does not hold.

Example $1(\mathrm{M} / \mathrm{D} / 1 / \infty)$ Consider a FIFO work-conserving server with service rate $r$ and infinite buffer capacity. Clearly, this system is a GR node with rate $r$ and latency $e=0$. The packet arrival process is Poisson with intensity $\lambda$ and packets are unit-length. For stability, we require $\lambda<r$.

We first show that $A$ is an EBB process. Note that $\mathrm{E}\left[e^{\theta A[0, t]}\right]=e^{\lambda t\left(e^{\theta}-1\right)}$. It is routinely observed that, for $\lambda<r$, $\mathrm{E}\left[e^{\theta A[0, t]}\right] \leq e^{r t \theta}$, for any $\theta \in\left[0, \theta_{0}\right]$, where $\theta_{0}$ is the unique strictly positive solution of $e^{\theta_{0}}-1=\frac{1}{\rho} \theta_{0}$, and $\rho:=\lambda / r$, the load of the system. By Chernoff's bound, one then obtains that $A$ is $\left(r, 1, \theta_{0}\right)$-EBB, i.e., for any $t \geq 0$,

$$
\mathrm{P}(A[0, t]-r t \geq \sigma) \leq e^{-\theta_{0} \sigma} .
$$

Let $D_{0}$ be the delay incurred by an arbitrary packet labeled with 0 . Then, we should be able to apply the result in [1], which, here, translates to:

$$
\mathrm{P}_{N}^{0}\left(D_{0} \geq u\right) \leq e^{-\theta_{0} r u}
$$

Next, we directly compute the delay distribution and match it against the hypothetical bound in (5). Let $V(t)$ be the unfinished work at $t$. Then, we know (see for instance [7], Sec. 6.1.1, p. 112), for $m \in \mathbb{Z}^{+}$,

$$
\mathrm{P}(V(0)>m)=1-(1-\rho) \sum_{n=0}^{m} \frac{[\rho(n-m)]^{n}}{n !} e^{-\rho(n-m)} .
$$

We consider the event $\left\{D_{0} \geq u\right\}$ for $u$ such that $r u$ is an integer. (Hence, $u=1 / r, 2 / r, \ldots$.) We next show that

$$
\mathrm{P}_{N}^{0}\left(D_{0} \geq u\right)=\mathrm{P}(V(0)>r u-1) .
$$

Note that given that by assumption we have fixed unit-length packets, there is no difference between $\mathrm{P}_{A}^{0}$ and $\mathrm{P}_{N}^{0}$. Hence, $\mathrm{P}_{N}^{0}\left(D_{0} \geq u\right)=\mathrm{P}_{N}^{0}(V(0) \geq r u)=\mathrm{P}_{A}^{0}(V(0) \geq r u)$. By PASTA ${ }^{2}$, we have $\mathrm{P}_{A}^{0}(V(0-) \geq r u)=\mathrm{P}(V(0) \geq r u)$. Now given that there is an arrival packet at $0, V(0)=V(0-)+$ 1. Hence, we obtain $\mathrm{P}_{A}^{0}(V(0) \geq r u)=\mathrm{P}(V(0) \geq r u-1)$. Putting the things together we recover the stated identity (7).

We can now do a direct evaluation of (7), using a numerical computation of (6), and compare it against $e^{-\theta_{0} r u}$, the upper bound predicted by (5). We see in Figure 1 that the bound does not hold, i.e. $\mathrm{P}_{N}^{0}\left(D_{0} \geq u\right)>e^{-\theta_{0} r u}$.

Comment. The example demonstrates that the probabilistic delay bound in [1] is incorrect. Notice that, in this example, given that the arrival process is Poisson, there is no bias due to the difference between Palm and stationary probabilities (PASTA). It will be seen later that when PASTA does not hold, we expect the delay bound to be even larger.

The rest of the note is organized as follows. In Section II we give a correct probabilistic bound on delay through an isolated GR node for an EBB arrival process. By known concatenation property, we extend the result to a sequence of GR nodes. In Section III we give fixes to the Theorems in [1] and [2].

\footnotetext{
${ }^{2}$ Poisson Arrivals See Time Averages; see, e.g., [6], Sec. 3.1, p. 165.
}

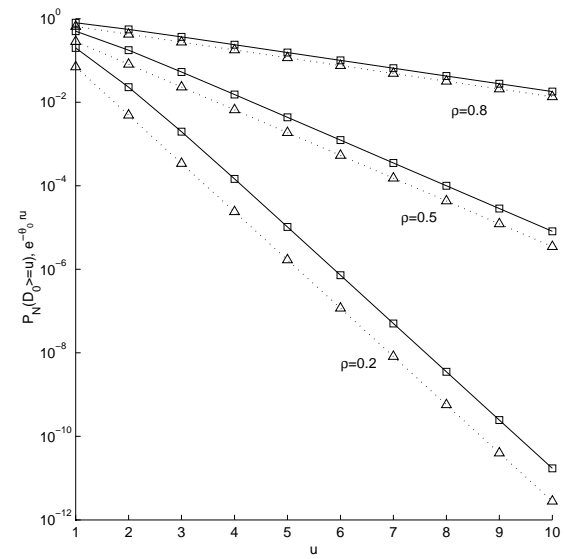

Fig. 1. Numerical values show (solid line) $\mathrm{P}_{N}^{0}\left(D_{0} \geq u\right)$ is greater than (dotted line) $e^{-\theta_{0} r u}(r$ is set to 1$)$.

\section{Probabilistic Bound on Delay for GR Nodes}

\section{A. Single Node Case}

Consider a single isolated GR node with rate $r$ and latency $e$. From the definition of GR node (1), it follows that for all $n \in \mathbb{Z}$,

$$
D_{n} \leq \max _{m \leq n}\left[\frac{A\left[T_{m}, T_{n}\right]}{r}-\left(T_{n}-T_{m}\right)\right]+e .
$$

Define $\tilde{V}(t)=\sup _{s<t}[A(s, t]-r(t-s)] . \quad \tilde{V}$ is the unfinished work of a hypothetical work-conserving constant rate server with rate $r$ that is fed with the same arrival process $A$ as our original system. Next, we show, for an arbitrary packet labeled with 0 , and $u \geq 0$, we have

$$
\mathrm{P}_{N}^{0}\left(D_{0} \geq u\right) \leq \frac{L^{\max }}{L^{\min }} \mathrm{P}_{A}^{0}(\tilde{V}(0) \geq r(u-e)) .
$$

Let $\lambda_{A}$ and $\lambda_{N}$ be the intensities of $A$ and $N . \quad\left(\lambda_{A}=\right.$ $\mathrm{E}[A(0,1]], \lambda_{N}=\mathrm{E}[N(0,1]]$.) By Campbell's formula [6], for some function $\psi$, and any $t \geq 0$,

$$
\begin{aligned}
& \mathrm{E}_{A}^{0}[\psi(\tilde{V}(0))]=\frac{1}{\lambda_{A} t} \mathrm{E}\left[\int_{0}^{t} \psi(\tilde{V}(s)) A(d s)\right] \\
& \mathrm{E}_{N}^{0}[\psi(\tilde{V}(0))]=\frac{1}{\lambda_{N} t} \mathrm{E}\left[\int_{0}^{t} \psi(\tilde{V}(s)) N(d s)\right] .
\end{aligned}
$$

From the last two identities, we conclude

$$
L^{\min } \mathrm{E}_{N}^{0}[\psi(\tilde{V}(0))] \leq \frac{\lambda_{A}}{\lambda_{N}} \mathrm{E}_{A}^{0}[\psi(\tilde{V}(0))] \leq L^{\max } \mathrm{E}_{N}^{0}[\psi(\tilde{V}(0))] .
$$

Now for $\psi=1$, from the last inequality we have $\lambda_{A} \leq$ $\lambda_{N} L^{\max }$, and hence

$$
\mathrm{E}_{N}^{0}[\psi(0)] \leq \frac{L^{\max }}{L^{\min }} \mathrm{E}_{A}^{0}[\psi(0)]
$$

From (8), we note $\mathrm{P}_{N}^{0}\left(D_{0} \geq u\right) \leq \mathrm{P}_{N}^{0}(\tilde{V}(0) \geq r(u-e))$. Finally, we apply (10) to the right-hand side of the last inequality to retrieve (9). Note that the ratio $L^{\max } / L^{\min }$ in (9) comes out with variable packet lengths and, clearly, it does not come into effect with fixed packet lengths.

Our aim is to obtain a bound on $\mathrm{P}_{N}^{0}\left(D_{0} \geq u\right)$ in terms of the stationary distribution of $\tilde{V}$. To that end, we use a corollary of 
Theorem 3 in [8] (distributional Little's law) that we pose as a lemma.

Lemma 1: Consider a work-conserving constant service rate server with rate $r$. The server is fed with packetized $A$ that is assumed to be stationary random measure with the intensity $\lambda_{A}<r$. $\left(\rho:=\lambda_{A} / r\right.$.) Then, for any non-decreasing measurable function $\psi: \mathbb{R}^{+} \rightarrow \mathbb{R}$

$$
\begin{aligned}
& (1-\rho) \psi(0)+\rho \mathrm{E}_{A}^{0}[\psi(\tilde{V}(0-))] \leq \mathrm{E}[\psi(\tilde{V}(0))] \leq \\
& \leq \rho \mathrm{E}_{A}^{0}[\psi(\tilde{V}(0))]+(1-\rho) \psi(0) .
\end{aligned}
$$

Note that $\tilde{V}(0)=\tilde{V}(0-)+A\{0\} \leq \tilde{V}(0-)+L^{\max }$. Hence, for $u \geq 0$,

$$
\mathrm{P}_{A}^{0}(\tilde{V}(0) \geq r(u-e)) \leq \mathrm{P}_{A}^{0}\left(\tilde{V}(0-) \geq r\left(u-e-\frac{L^{\max }}{r}\right)\right) .
$$

Now we apply Lemma 1 for $\psi(x)=1_{x \geq v}, v>0$, to obtain

$$
\mathrm{P}_{A}^{0}(\tilde{V}(0-) \geq v) \leq \frac{r}{\lambda_{A}} \mathrm{P}(\tilde{V}(0) \geq v) .
$$

From (9) and the last two displays, it follows, for $u>e+\frac{L^{\max }}{r}$,

$$
\mathrm{P}_{N}^{0}\left(D_{0} \geq u\right) \leq \frac{L^{\max }}{L^{\min }} \frac{r}{\lambda_{A}} \mathrm{P}\left(\tilde{V}(0) \geq r\left(u-e-\frac{L^{\max }}{r}\right)\right) .
$$

We next show the main result of this section - probabilistic bound on delay through a GR node for EBB arrival process.

Theorem 1: Consider a GR node with rate $r$ and latency $e$. The node is fed with stationary random $A$ of intensity $\lambda_{A}$; in addition, $A$ is $(\lambda, C, c)$-EBB with $\lambda<r$. If time is continuous, then, for $u>0$,

$$
\mathrm{P}_{N}^{0}\left(D_{0} \geq u+e+\frac{L^{\max }}{r}\right) \leq \frac{L^{\max }}{L^{\min }} \frac{r}{\lambda_{A}} \frac{C e^{c \lambda \delta}}{1-e^{-c(r-\lambda) \delta}} e^{-c r u},
$$

where $\delta>0$ is such that $\frac{C e^{c \lambda \delta}}{1-e^{-c(r-\lambda) \delta}} e^{-c r \delta} \geq 1$. If time is discrete, then replace the right-hand side in (12) with

$$
\frac{L^{\max }}{L^{\min }} \frac{r}{\lambda_{A}} \frac{C}{1-e^{-c(r-\lambda)}} e^{-c r u} .
$$

Notice that the assumption that the arrival process $A$ is $(\lambda, C, c)$ EBB implies that $\lambda_{A} \leq \lambda[4]$.

Comment. We first compare (12) with [4]. The bounds in [4] are for the unfinished work (divided by $r$, it corresponds to the virtual waiting time) of a work-conserving constant service rate server; they are for the steady-state probability $P$. Their validity requires only to assume that $A$ is with $\mathrm{EBB}$; there is no need to assume that $A$ is stationary. In contrast, for the bound on the waiting time distribution given here (which is for the Palm probability $\mathrm{P}_{N}^{0}$ ), we need to assume that $A$ is stationary, in order to apply the result of [8].

Next, we discuss how (12) differs from Theorem 4 in [1] (equation (46) therein). The discussion is for an isolated GR node; we later give extension for the delay through a sequence of nodes. Note that we have an additional pre-factor and the latency term $L^{\max } / r$. In total, we expect (12) to be larger than the (incorrect) bound in [1].

Proof: The proof follows from (11) and a known bound for the unfinished work of a constant rate server with rate $r$ and EBB arrival process (see. Equation (4) in [4]). Equation (13) is proved likewise (see remark to Theorem 1 in [4]).

\section{B. End-to-end Delay Bound}

Consider a sequence of $h$ GR nodes with rates and latencies $\left(r_{l}, e_{l}\right)_{l=1}^{h}$. It is known that this concatenation is a GR node with rate $r=\min _{1 \leq l \leq h} r_{l}$ and latency $e=\sum_{l=1}^{h} e_{l}+L^{\max } \sum_{l=1}^{h-1} \frac{1}{r_{l}}$ ([1]; see also Sec. 2.1.3 [5]). Suppose the sequence of GR nodes is fed with a $(\lambda, C, c)$-EBB arrival process $A$ with $\lambda<r$. Then, a probabilistic bound on the end-to-end delay is obtained from Theorem 1 by replacing $r$ and $e$ as defined in this section.

\section{Correction of the Theorems in [1] and [2]}

We first give a correct version of Theorem 4 in [1].

Theorem 2: If flow $f$, with stationary random arrival process $A$, conforms to EBB with parameters $\left(\lambda^{f}, \Lambda^{f}, \gamma^{f}\right)$, has intensity $\lambda_{A}^{f}$, and the scheduling algorithm at each of the servers on the path of a flow belongs to GR for the flow (rate $r^{f}$ and latency $e_{l}^{f}, l=1, \ldots, h$, such that $\left.\lambda^{f}<r^{f}\right)$, then the end-to-end delay of packet 0 denoted as $D_{0}^{f}$ is given by, for $u>0$ :

$$
\mathrm{P}_{N}^{0}\left(D_{0}^{f} \geq u+e^{f}\right) \leq \frac{L^{\max }}{L^{\min }} \frac{r^{f}}{\lambda_{A}^{f}} \frac{\Lambda^{f} e^{\gamma^{f} \rho^{f} \delta}}{1-e^{-\gamma^{f}\left(r^{f}-\rho^{f}\right) \delta}} e^{-\gamma^{f} r^{f} u}
$$

where, for brevity, $e^{f}:=h \frac{L^{\max }}{r^{f}}+\sum_{l=1}^{h} e_{l}^{f}$, and $\delta>0$ is such that $\frac{\Lambda^{f} e^{\gamma^{f} \lambda^{f} \delta}}{1-e^{-\gamma_{f}\left(r^{f}-\lambda^{f}\right) \delta}} e^{-\gamma^{f} r^{f} \delta} \geq 1$. Here $L^{\min }$ and $L^{\text {max }}$ are the minimum and maximum packet lengths of the flow $f$.

Comment. Note that in contrast to [1], we require $\lambda^{f}<r^{f}$ to ensure stability (in [1], $\lambda^{f}=r^{f}$ ).

Next we give fixes to Theorem 2 and Theorem 4 in [2]. The fixes are merely some appropriate substitutions of the rate and latency parameters in our Theorem 2 . In Theorem 2 [2] use Theorem 2 above with the latency parameters defined as in Lemma 3 [2] (resp. for Theorem 4 [2] as defined in Lemma 5 [2]).

\section{Acknowledgments}

We thank to one of the anonymous reviewers for pointing them to two errors in a preliminary draft of the paper.

\section{References}

[1] P. Goyal, S. S. Lam, and H. M. Vin, "Determining end-to-end delay bounds in heterogeneous networks," Springer Multimedia Systems, vol. 5, pp. 157$163,1997$.

[2] B. Bensaou, D. H. K. Tsang, and K. T. Chan, "Credit-based fair queueing (CBFQ): A simple service-scheduling algorithm for packet-switched networks," IEEE/ACM Trans. on Networking, vol. 9, no. 5, pp. 591-603, October 2001.

[3] P. Goyal, S. S. Lam, and H. M. Vin, "Determining end-to-end delay bounds in heterogeneous networks," in Proc. 5th Int. Workshop Network and Operating System Support for Digital Audio and Video, April 1995, pp. 287-298.

[4] O. Yaron and M. Sidi, "Performance and stability of communication networks via robust exponential bounds," IEEE/ACM Trans. on Networking, vol. 1, no. 3, pp. 372-384, June 1993.

[5] J.-Y. Le Boudec and P. Thiran, Network Calculus, Springer-Verlag, 2001.

[6] F. Baccelli and P. Brémaud, Elements of Queueing Theory, vol. 26, Applications of Mathematics, Springer-Verlag, 1991.

[7] J. W. Roberts (Editor), COST 224: Performance evaluation and design of multiservice networks, Commission of the European Communities, 1991.

[8] T. Konstantopoulos and G. Last, "On the dynamics and performance of stochastic fluid systems," Journal of Applied Probability, vol. 37, pp. 652$667,2000$. 